\title{
Retrospective study in 608 cases on the rate of surgical site infections after orbital surgery without prophylactic systemic antibiotics
}

\author{
Ronald Olaf Björn de Keizer, Genesis Kozdras, René Wubbels, Willem A Van den Bosch, \\ Dion Paridaens
}

Oculoplastic and Orbital Service, The Rotterdam Eye Hospital, Rotterdam, The Netherlands

\section{Correspondence to}

Ronald Olaf Björn de Keizer, The Rotterdam Eye Hospital Oculoplastic and Orbital Service, Rotterdam, The Netherlands; $r$. dekeizer@oogziekenhuis.nl

Received 25 March 2018 Revised 21 November 2018 Accepted 26 November 2018 Published Online First 21 December 2018
Check for updates

(C) Author(s) (or their employer(s)) 2019. No commercial re-use. See rights and permissions. Published by BMJ.

To cite: de Keizer ROB Kozdras G, Wubbels R, et al. $\mathrm{Br} J$ Ophthalmol

2019:103:1466-1468.

\section{ABSTRACT}

Objective To determine postoperative surgical site infection (SSI) rates in three separate patient groups who underwent orbital surgery without prophylactic systemic antibiotics.

Study design Single-centre retrospective descriptive

case series.

Study population We studied the notes of 639 consecutive patients who had undergone orbital surgery in our hospital from 2009 through 2013. All patients belonged to either of three groups: (1) clean orbital surgery ( $n=226) ;(2)$ clean orbital surgery with implant $(n=290) ;(3)$ clean-contaminated surgery $(n=92)$. Thirtyone patients were excluded.

Results Of the total of 608 patients, without systemic antibiotic prophylaxis, only five were diagnosed with SSI 5/608 (0.82\%): 1/226 in the 'clean' group, 3/290 in the 'clean-with-implant' group and 1/92 in the 'cleancontaminated' group. All five patients with SSI were effectively treated with antibiotics.

Conclusion In this study 'clean', 'clean-with-implant' and 'clean-contaminated' orbital surgery was safely performed without prophylactic antibiotics. Where postoperative infection did occur, the patients were effectively treated with systemic antibiotics. We suggest to restrict the administration of systemic antibiotic prophylaxis in orbital surgery.

\section{INTRODUCTION}

Recently, WHO advised to minimise the use of antibiotics because of increasing resistance and lack of new generations of antibiotics. ${ }^{1}$ However, antibiotic drugs continue to be widely used both for prophylaxis and treatment of infection before, during or after surgical procedures, while standards of perioperative care regarding treatment regimens vary between countries. The literature about the use of antibiotics in oculoplastic and orbital surgery is scarce. $^{2}$

Recent retrospective studies about eviscerations and enucleations show that withholding antibiotic prophylaxis from these patients did not increase the number of surgical site infections (SSIs).$^{2-4}$ Similarly, a recent retrospective study from our group did not show any SSI in 186 enucleations (without prophylactic antibiotics) by a single surgeon. ${ }^{5}$ We are not aware of studies about the prophylactic antibiotics use in other intraorbital surgical procedures.

In the National Institute for Health and Care Excellence update (February 2017) on (general)
SSIs, it was advised to provide prophylactic antibiotics in clean-contaminated and in clean surgery with implants or prosthesis, by giving a single dose of intravenous antibiotics prior to incision on starting anaesthesia. ${ }^{4}$ In our hospital, however, we have not routinely used prophylactic systemic antibiotics in orbital surgery for clean, clean with implant or clean-contaminated surgery. The only indications in our institute for preoperative or perioperative systemic antibiotics are severely immunocompromised patients and/or patients with an active infection such as endophthalmitis with corneal perforation, orbital cellulitis or patients with increased risk of endocarditis.

In this study, we evaluate the postoperative infection rate of orbital surgery without prophylactic systemic antibiotics in a retrospective case series.

\section{METHODS}

We retrospectively analysed the records of all patients who underwent orbital surgery in our hospital between 2009 and 2013. We included patients who underwent orbital decompression, orbitotomy for tumour excision, enucleation, evisceration, exenteration, orbital biopsy and other orbital (reconstructive) surgery. Patients required a follow-up of at least 30 days after the procedure. Patients were categorised in three groups according to the type of orbital surgery: 'clean' orbital surgery, 'clean-with-implant' orbital surgery and 'clean-contaminated' orbital surgery. Of each patient we recorded the age, gender, American Society of Anesthesiologists (ASA) classification, type of surgery, use of systemic antibiotics and occurrence of postoperative SSI.

\section{DEFINITIONS}

- Clean: an uninfected operative wound in which no inflammation is encountered, and the respiratory tracts are not entered.

- Clean with implant: as stated, but with implant of an allogenic material.

- Clean-contaminated: operative wounds in which the respiratory tracts are entered under controlled conditions and without unusual contamination, provided no evidence of infection or major break in technique is encountered.

- SSI is defined as an event within 30 days after the orbital surgical procedure. We retrospectively categorised to the Centers for Disease Control and Prevention (CDC) definitions. This 
Table 1 Characteristics of the patient groups studied

\begin{tabular}{|c|c|c|c|c|}
\hline & $\begin{array}{l}\text { Clean } \\
\mathrm{N}\end{array}$ & $\begin{array}{l}\text { Clean with } \\
\text { implant } \\
\mathrm{N}\end{array}$ & $\begin{array}{l}\text { Clean- } \\
\text { contaminated } \\
\mathrm{N}\end{array}$ & Total \\
\hline Mean age at surgery (range) & $54(1-87)$ & $58(4-98)$ & $50(3-87)$ & \\
\hline \multicolumn{5}{|l|}{ Gender } \\
\hline Male & 108 & 152 & 20 & 280 \\
\hline Female & 118 & 138 & 72 & 328 \\
\hline \multicolumn{5}{|l|}{ ASA classification } \\
\hline No of patients $\leq 2$ & 198 & 233 & 82 & 513 \\
\hline No of patients $>2$ & 28 & 57 & 10 & 95 \\
\hline Total & 226 & 290 & 92 & 608 \\
\hline \multicolumn{5}{|l|}{ Surgery } \\
\hline Biopsy & 71 & & & \\
\hline Debulking & 18 & & & \\
\hline Tumour excision & 96 & & & \\
\hline Decompression* & 11 & & 92 & \\
\hline Exenteration & 6 & & & \\
\hline Socket reconstructiont & 17 & 57 & & \\
\hline Other & 7 & & & \\
\hline Evisceration & & 159 & & \\
\hline Enucleation & & 74 & & \\
\hline Total & 226 & 290 & 92 & 608 \\
\hline \multicolumn{5}{|l|}{ Excluded patients } \\
\hline Due to prophylaxis & 3 & 7 & 3 & 13 \\
\hline $\begin{array}{l}\text { Due to preoperative } \\
\text { infection }\end{array}$ & 4 & 8 & 6 & 18 \\
\hline Total & 7 & 15 & 9 & 31 \\
\hline
\end{tabular}

*Eleven patients ( 6 bilateral) had solely lateral wall decompression and 93 (76 bilateral) had decompression with an opening to the nose or one of the sinuses. tSeventeen patients had socket reconstruction without an alloplastic implant, 57 with alloplastic implant.

ASA, American Society of Anesthesiologists.

resulted in wound infections as a superficial SSI, preseptal cellulitis as deep incisional SSI and orbital cellulitis as organ/ space SSI. ${ }^{6}$

- ASA physical status: assessment by the anaesthesiologist of the patient's preoperative physical condition using the ASA Classification of Physical Status. ${ }^{6}$

All patients with a contaminated, dirty or infected orbital disease were excluded. All patients having antibiotic prophylaxis in any form were excluded. We examined the records of 639 patients; 608 of these did not have systemic antibiotics prior to surgery, while 31 had received antibiotic treatment or had a dirty wound and were excluded.

\section{RESULTS}

Table 1 shows the patient characteristics, including age, gender, ASA classification and type of surgery in each study group.

Table 2 shows the incidence of SSI in the three patient groups and the ASA classification.

Infection of the surgical site occurred in one patient in the 'clean' group, three in the 'clean with implant' group and one in the 'clean-contaminated' group. SSI occurred more frequently in ASA $\leq 2$ patients $(n=5 / 508)$ than in ASA $>2$ patients $(0 / 95)$. A group of 31 patients was treated with antibiotics preoperatively: 13 with systemic antibiotic prophylaxis and 18 with antibiotics as therapy for underlying disease; some had prolonged therapy after endophthalmitis.

The patients with a SSI are shown in table 3.
Table 2 Outcome of SSIs found in the three studied patient groups

\begin{tabular}{|c|c|c|c|c|}
\hline & $\begin{array}{l}\text { Clean } \\
\mathrm{N}\end{array}$ & $\begin{array}{l}\text { Clean } \\
\text { with } \\
\text { implant } \\
\mathrm{N}\end{array}$ & $\begin{array}{l}\text { Clean- } \\
\text { conta } \\
\text { minated } \\
\mathrm{N}\end{array}$ & Total \\
\hline \multicolumn{5}{|l|}{ SSI in ASA groups } \\
\hline No of patients ASA $\leq 2$ without SSI & 197 & 230 & 81 & 508 \\
\hline No of patients ASA $\leq 2$ with SSI & 1 & 3 & 1 & 5 \\
\hline No of patients $>2$ without SSI & 28 & 57 & 10 & 95 \\
\hline No of patients ASA $>2$ with SSI & 0 & 0 & 0 & 0 \\
\hline Total & 226 & 290 & 92 & 608 \\
\hline
\end{tabular}

ASA, American Society of Anesthesiologists; SSI, surgical site infection.

Of the five patients with clinical signs suggestive of infection, two had been clinically diagnosed with wound infection, two with preseptal orbital cellulitis and one with all the signs of an orbital cellulitis.

Two other patients from the group of 608 patients did not show signs of infection, but their general physicians had started oral antibiotics without consulting us because of 'bloody discharge' after a medial wall decompression and because of 'conjunctival swelling' after orbital surgery, respectively. We therefore considered these two cases as not having a surgical site infection.

\section{DISCUSSION}

This study is limited by the retrospective design, the low number of patients per treatment group and the heterogeneity of the procedures. We cannot exclude the possibility that, due to the low rate of SSI as observed in the study, this is explained by chance alone, but our results indicate that orbital surgery without the use of prophylactic antibiotic therapy is rarely complicated by SSIs. All patients who exhibited SSI responded well to subsequent administration of antibiotics.

Since the late 1980s, no new antibiotics have been discovered (the 'Discovery Void') while drug resistance is increasing. ${ }^{17}$ Therefore, the need to control the use of antibiotics becomes more and more important. Apart from resistance increase, antibiotic use is associated with a wide variety of side effects and complications such as anaphylaxis, Stephens-Johnson syndrome, gastrointestinal distress, nephrotoxicity, ototoxicity, hepatic toxicity, cardiac toxicity, renal insufficiency, neutropenia, neuritis, myelosuppression, vaginitis, sun sensitivity, and allergic response. Antibiotics may also interact unfavourably with other medication. $^{38}$

In 2005, results were published of a prospective randomised trial regarding the efficacy of adjuvant oral antibiotics preoperative next to one dose of systemic perioperative prophylactic antibiotics in colorectal surgery. ${ }^{9}$ No difference in SSIs was found between groups, while antibiotic use was associated with an increase in side effects, such as postoperative vomiting, nausea and abdominal pain. The side effects were significantly lower in the group of patients who did not receive any adjuvant oral preoperative antibiotics. $^{9}$

The effect of postoperative antibiotic prophylaxis has been studied in a retrospective, multicentre, comparative case series. ${ }^{3}$ Of 644 eye eviscerations or enucleations, 381 had been treated with postoperative antibiotic prophylaxis and 263 had not. In the first group, one presumed case of orbital cellulitis had occurred $(0.26 \%)$ and also one $(0.38 \%)$ in the second group. Both groups received one dose of perioperative antibiotic prophylaxis. A recent study of Pariseau compared 480 cases of enucleation and evisceration: in the group of 70 patients with 
Tabel 3 SSI in 608 patients after orbital surgery without antibiotic prophylaxis

\begin{tabular}{|c|c|c|c|c|c|c|c|c|c|}
\hline Patient & Age & Gender & ASA & Surgery & Group & SSI & $\begin{array}{l}\text { Days after } \\
\text { surgery }\end{array}$ & Treatment & Culture \\
\hline 1 & 39 & $\mathrm{~F}$ & 1 & $\begin{array}{l}\text { Lateral orbitotomy for cavernous } \\
\text { hemangioma }\end{array}$ & $\mathrm{cl}$ & Wound infection & 2 & Oral amoxicillin/clavulanic acid & None \\
\hline 2 & 59 & $\mathrm{~F}$ & 1 & $\begin{array}{l}\text { Evisceration for painful blind eye; acrylic } \\
\text { implant }\end{array}$ & $c+i$ & Orbital cellulitis & 3 & $\begin{array}{l}\text { Intravenous amoxicillin/ } \\
\text { clavulanic acid; oral } \\
\text { clindamycin }\end{array}$ & $\begin{array}{l}\text { Staphylococcus } \\
\text { aureus }\end{array}$ \\
\hline 3 & 34 & M & 1 & Evisceration after trauma; acrylic implant & $c+i$ & Preseptal cellulitis & 4 & Oral amoxicillin/clavulanic acid & Culture negative \\
\hline 4 & 65 & M & 2 & $\begin{array}{l}\text { Evisceration after complications of } \\
\text { intraoculur surgery; acrylic implant }\end{array}$ & $\mathrm{c}+\mathrm{i}$ & Preseptal cellulitis & 5 & Oral azithromycin & None \\
\hline 5 & 80 & $\mathrm{~F}$ & 2 & $\begin{array}{l}\text { Orbital decompression medial wall for } \\
\text { dysthyoid opticoneuropathy }\end{array}$ & $\mathrm{CC}$ & Wound infection & 7 & Oral amoxicillin/clavulanic acid & None \\
\hline
\end{tabular}

Patient 6 got preoperative antibiotic prophylaxis, amoxicillin/clavulanic acid and was excluded from the study.

ASA, American Society of Anesthesiologists; SSI, surgical site infection; cc, clean-contaminated; c+i, clean with implant; cl, clean.

perioperative intravenous antibiotics, the SSI rate was $1.4 \%$; and in the group of 411 cases not given perioperative intravenous antibiotics, the SSI rate was $1.1 \%$ for eviscerations with implants and $0.7 \%$ for enucleations with implants. In the systematic review in the same paper, the SSI rate in other studies about evisceration was between $0.6 \%$ and $7 \%$, and between $0 \%$ and $13 \%$ for enucleation. ${ }^{2}$

Several other studies on prophylactic (preoperative or postoperative) antibiotics regarding surgery of the jaws, ears and dental surgery also failed to show evidence for antibiotic prophylaxis to prevent SSIs. ${ }^{1011}$

In our single-centre retrospective case series without systemic prophylactic antibiotic treatment, patients with almost all types of orbital surgery were included. The rate of SSI proved comparable with those reported by other studies, with and without preoperative and/or perioperative prophylactic antibiotics. ${ }^{23}$

We prescribe postoperative systemic antibiotics (usually a combination of oral amoxicillin and clavulanate) only in case of evident or suspected wound infection (deep or superficial) or evident or suspected orbital cellulitis (organ space as defined by the CDC). ${ }^{12}$ We feel there is no conclusive evidence that demonstrates the usefulness of systemic antibiotics as a prophylaxis in orbital surgery, while it is known that adverse side effects occur relatively frequently.

In our series, 'clean', 'clean surgery with implant' and 'clean-contaminated' surgery in the absence of prophylactic systemic antibiotic treatment could be compared. No substantial differences with respect to postoperative infection rate were found, although numbers per group were low, and the groups itself are heterogenous and therefore limiting the conclusions to be drawn.

In conclusion, our retrospective series shows that orbital surgery may be performed safely without prophylactic systemic antibiotics. The small proportion of cases $(0.82 \%)$ with presumed signs of postoperative infection could be treated effectively with standard antibiotics. We have restricted the use of preoperative and perioperative systemic prophylactic antibiotics to severely immunocompromised patients. This, however, also requires further study, preferably through a prospective trial.
Contributors All authors substantially contributed to the conception or design of the work, or the acquisition, analysis or interpretation of data; drafted the work or revised it critically for important intellectual content; and approved the final version published.

Competing interests None declared.

Patient consent for publication Not required.

Ethics approval Approval was obtained from the scientific review board of the Rotterdam Eye Hospital/Rotterdam Ophthalmic Institute.

Provenance and peer review Not commissioned; externally peer reviewed.

Data sharing statement Individual participant data that underlie the results reported in this article, after deidentification. This is including the data of the excluded patients. Interested orbital surgeons can request the anonymised data by mail, when a methodologically sound proposal is provided and has been approved by our independent review committee. Proposals should be directed to r.dekeizer@ oogziekenhuis.nl. To gain access, data requestors will need to sign a data access agreement.

\section{REFERENCES}

1 WHO. The evolving threat of antimicrobial resistance Options for action. 2012. Available: http://apps.who.int/iris/bitstream/10665/44812/1/9789241503181_eng. pdf [Accessed 20 Oct 2016].

2 Pariseau B, Fox B, Dutton JJ. Prophylactic antibiotics for enucleation and evisceration: a retrospective study and systematic literature review. Ophthalmic Plast Reconstr Surg 2018;34:49-54.

3 Fay A, Nallasamy N, Nallassamy N, et al. Prophylactic postoperative antibiotics for enucleation and evisceration. Ophthalmic Plast Reconstr Surg 2013:29:281-5.

4 Nice. Surgical site infection Evidence Update February 2017. 2018. Available: www. nice.org.uk [Accessed 17 Nov 2018].

5 Verhoekx JSN, Tse WHW, Rengifo Coolman A, et al. Complications following enucleations and subsequent oculoplastic surgeries. Ophthalmic Plast Reconstr Surg 2018:34:320-3.

6 CDC. Procedure-associated module January 2018. 2018. Available: https://www.cdc. gov/nhsn/pdfs/pscmanual/9pscssicurrent.pdf [Accessed 17 Nov 2018].

7 Zorzet A. Overcoming scientific and structural bottlenecks in antibacterial discovery and development. Ups J Med Sci 2014:119:170-5.

8 Richardson WL, Hammert WC. Adverse effects of common oral antibiotics. J Hand Surg Am 2014;39:989-91.

9 Espin-Basany E, Sanchez-Garcia JL, Lopez-Cano M, et al. Prospective, randomised study on antibiotic prophylaxis in colorectal surgery. Is it really necessary to use oral antibiotics? Int J Colorectal Dis 2005;20:542-6.

10 Brignardello-Petersen R, Carrasco-Labra A, Araya I, et al. Antibiotic prophylaxis for preventing infectious complications in orthognathic surgery. Cochrane Database Syst Rev 2015;1:CD010266

11 Verschuur HP, de Wever W, van Benthem PP, et al. Antibiotic prophylaxis in clean and clean-contaminated ear surgery. Cochrane Database Syst Rev 2004;8.

12 Mangram AJ, Horan TC, Pearson ML, et al. Guideline for prevention of surgical site infection, 1999. Infect Control Hosp Epidemiol 1999;20:247-80. 